\title{
EchoGéo
}

\section{Acquisitions de la Bibliothèque de l'Institut de Géographie}

\section{Bernadette Joseph}

\section{(2) OpenEdition}

1 Journals

\section{Édition électronique}

URL : https://journals.openedition.org/echogeo/11838

DOI : $10.4000 /$ echogeo. 11838

ISSN : 1963-1197

\section{Éditeur}

Pôle de recherche pour l'organisation et la diffusion de l'information géographique (CNRS UMR 8586)

Référence électronique

Bernadette Joseph, « Acquisitions de la Bibliothèque de l'Institut de Géographie », EchoGéo [En ligne], 12 | 2010, mis en ligne le 31 mai 2010, consulté le 31 juillet 2021. URL : http:// journals.openedition.org/echogeo/11838; DOI : https://doi.org/10.4000/echogeo.11838

Ce document a été généré automatiquement le 31 juillet 2021.

EchoGéo est mis à disposition selon les termes de la licence Creative Commons Attribution - Pas d'Utilisation Commerciale - Pas de Modification 4.0 International (CC BY-NC-ND) 


\title{
Acquisitions de la Bibliothèque de l'Institut de Géographie
}

\author{
Bernadette Joseph
}

\section{NOTE DE L'ÉDITEUR}

Certains titres présents dans cette liste ne correspondent pas obligatoirement à une nouvelle acquisition du titre mais à l'acquisition d'exemplaires supplémentaires.

\section{Afrique}

1 ARTUSO, Décentralisation au Niger: résultats et questions ouvertes, Paris, L'Harmattan, 2009, G 19-3518

2 ATTENOUKON, L'Afrique, poubelle de l'Occident ?: la gestion des déchets dangereux, Paris, L'Harmattan, 2009, G 19-3516

3 BAKOUP, BOISSIEU, HENNER, L'Afrique peut-elle gagner sa place dans la mondialisation? Pour une politique économique systémique, Paris, L'Harmattan, 2009, G 19-3511

BIYOYA MAKUTU KAHANDJA, La géopolitique de l'instabilité dans la région des Grands lacs : réflexions sur les réfugiés, ces acteurs-auteurs des mutations géostratégiques, Paris, L'Harmattan, 2009, G 19-3508 BOSSARD, Atlas régional de l'Afrique de l'Ouest, Paris, OCDE ; CSAO, 2009, G 19-3513

BRACHET, Migrations transsahariennes: vers un désert cosmopolite et morcelé (Niger), Bellecombe-en-Bauges, Ed. du Croquant, 2009, G 19-3512 COMBIER, Carte géologique de Dakar, Paris, Larose, 1935, G 19-3522 DUBOIS, La vie au continent noir, Paris, J. Hetzel, 1893, G 19-3520

9 FWELEY DIANGITUKWA, Les grandes puissances et le pétrole africain : États-Unis - Chine, une compétition larvée pour l'hégémonie planétaire, Paris, L'Harmattan, 2009, G 19-3525 
GERVAIS-LAMBONY, L'Afrique du Sud, Paris, Le Cavalier bleu, 2009, G 19-3509

GRAS, JAMATI, Voyage en Afrique urbaine: urbanisation, modernité et société, Paris, L'Harmattan, 2009, G 19-3514

HIRSCH, ROUSSEL, COPPENS, Le rift est-africain: une singularité plurielle, Marseille, IRD éd. ; Paris, Publications scientifiques du Muséum, 2009, G 19-3507

JOLY, Guerres d'Afrique : 130 ans de guerres coloniales : l'expérience française, Rennes, Presses universitaires de Rennes, 2009, G 19-3519

LORY, L'Afrique du sud, Paris, Éd. Karthala, 2010, G 19-3526

MAUPOIL, Coutumiers juridiques de l'Afrique occidentale française, Paris, Larose, 1939, G 19-3521

MOHAMED GHADHI, La longue marche de l'Afrique vers l'intégration, le développement et la modernité politique, Paris, L'Harmattan, 2009, G 19-3510

SADIGH, Afrique, le continent pillé: atouts, handicaps, perspectives et propositions, Paris, L'Harmattan, 2009, G 19-3517

SASSOU NGUESSO, L'Afrique, enjeu de la planète: les nouveaux défis du développement durable, Gémenos, Respublica ; J-M. Laffont, 2009, G 19-3515

SOKO, Les modèles de microfinance en Côte d'Ivoire : origine, organisation et impact, Paris, L'Harmattan, 2009, G 19-3523

TCHIBAMBELELA, Le commerce mondial de la faim : stratégie de rupture positive au CongoBrazzaville, Paris, L'Harmattan, 2009, G 19-3524

1 TEULIÉ, Healing South African Wounds, Montpellier, Presses universitaires de la Méditerranée, 2009, G 19-3527

\section{Amérique du nord, Arctique}

FIREY, Land use in central Boston, Cambridge, Harvard University Press, 1947, G 20-1645

MAILLARD, Circulation des cerveaux entre la Chine et la Californie: étudiants, ingénieurs, entrepreneurs et capital-risqueurs chinois dans la Silicon Valley, Paris, L'Harmattan, 2009, G 20-1642

MASSICOTTTE, Sciences du territoire: perspectives québécoises, Québec, Presses de l'Université du Québec, 2008, G 20-1643

PARKINS, The South : its economic-geographic development, New York ; London, J. Wiley ; Chapman \& Hall, 1938, G 20-1644

Quel avenir pour le Groenland ?, Paris, Choiseul, 2009, G 20-1641

SCHOR, Compter et classer : histoire des recensements américains, Paris, Éd. de l'École des hautes études en sciences sociales, 2009, G 20-1646

THEVENET, Le Groenland : Kalaallit Nunaat, Paris, Karthala, 2009, G 20-1640

\section{Amérique latine}

Aspects environnementaux en Amérique Latine, Pessac, Presses universitaires de Bordeaux, 2009, G 21-1711 

21-1710

\section{Asie, Océanie} 2009, G 17-2720 G 17-2721 G 17-2725

BABOU, Disposer de la nature: enjeux environnementaux en Patagonie argentine, Paris, L'Harmattan, 2009, G 21-1709

BITÉGUÉ DIT MANGA, Lecture publique et identités locales : le cas des territoires isolés de l'intérieur de la Guyane, Matoury (Guyane), Ibis rouge éd., 2010, G 21-1717

BOISARD, ENDERS, VERDO, L'Amérique latine des régimes militaires, Paris, Presses de la Fondation nationale des sciences politiques, 2010, G 21-1716

DELER, El manejo del espacio en el Ecuador: etapas claves, Quito, Centro Ecuatoriano de Investigación Geográfica, 1983, G 21-1713

GIRAULT, Intégrations en Amérique du sud, Paris, Presses Sorbonne nouvelle, 2009, G

GRAVEL, Géographie de l'Amérique latine: une culture de l'incertitude, Québec, Presses de l'Université du Québec, 2009, G 21-1714

GREGORY, The devil behind the mirror : globalization and politics in the Dominican Republic, Berkeley, University of California Press, 2007, G 21-1712

HUME, The politics of violence : gender, conflict and community in El Salvador, Malden, Wiley-Blackwell, 2009, G 21-1708

MARTINEZ, Diccionario geográfico del Estado Táchira, Mérida (Venezuela), Universidad de Los Andes, 1962, G 21-1715

BAIXAS, DEJOUHANET, TROUILLET, Conflit et rapports sociaux en Asie du Sud, Paris, L'Harmattan, 2010, G 17-2732

BAMBRIDGE, La terre dans l'archipel des Australes : étude du pluralisme juridique et culturel en matière foncière (Pacifique Sud), Pirae (Tahiti), Au vent des îles ; Arue (Tahiti), IRD éd.,

BERTUZZO, Fragmented Dhaka : analysing everyday life with Henri Lefebvre's theory of production of space, Stuttgart, F. Steiner, 2009, G 17-2726

CONNELL, RUGENDYKE,Tourism at the grassroots : villagers and visitors in the Asia-Pacific, London; New York, Routledge, 2008, G 17-2723

COURMONT, Chine, la grande séduction : essai sur le soft power chinois, Paris, Choiseul, 2009,

DUCOURTIEUX, Du riz et des arbres: l'interdiction de l'agriculture d'abattis-brûlis, une constante politique au Laos, Paris ; Marseille, Karthala ; IRD, 2009, G 17-2724

DUPONT, LANDY, Circulation et territoire dans le monde indien contemporain, Paris, Éd. de l'École des hautes études en sciences sociales, 2010, G 17-2731

GANGULI, Trends of agriculture and population in the Ganges valley : a study in agricultural economics, London, Methuen \& Co., 1938, G 17-2730

GILQUIN, Atlas des minorités musulmanes en Asie méridionale et orientale, Paris, CNRS, 2010,

LAUREY, Énergies renouvelables: plaidoyer pour une véritable politique de l'énergie en Polynésie française, Pirae (Tahiti), Aux vents des îles, 2009, G 17-2722 

l'espace Amazonie-Caraibes en quête d'un projet territorial, Paris, Éditions Cujas, 2009, G 3-3145.

\section{saoudien pour le dialogue des civilisations, Riyad, 6-10 mars 2009. Paris, Karthala Observatoire d'études géopolitiques, Université Paris Descartes, Faculté de droit, 2010,} G 3-3144

\section{Congrès}

61 ACKERMANN, Qu'est-ce qui construit l'aire urbaine ?: actes du séminaire, vendredi 24 novembre 2006, Atria, Belfort, Montbéliard, Syndicat mixte Aire urbaine Belfort Montbéliard Héricourt Delle, 2009, G 3-3134 


\section{Tschech 3136}

\section{Paris-Sorbonne, Paris, Université de Paris-Sorbonne, Service de reprographie, 1998, G} 3-3135

Formes urbaines du futur et mobilité non motorisée: la mobilité autogène, marche, bicyclette, roller... a-t-elle une place dans les villes du XXIe siècle? Actes du séminaire du 17 décembre 1999, Paris, Amarcande, 2000, G 3-3137 ruralité ? Université d'été, Ve édition, Erba, 27-28-29/08/2008 Como, Bruxelles, Bruylant, 2009, G 3-3122

77

AGUCHI, SHIMOKAWA, Japanese foreign direct investment and the East Asian industrial system : case studies from the automobile and electronics industries, Tokyo ; Berlin, Springer, 2002, G 3-3143

78 Opportunités et enjeux du développement de l'Afrique dans l'arène mondiale: actes de la conférence économique africaine 2007, Paris, Economica, 2009, G 3-3129

79 Programmer les logements: séance du 22 janvier 2008, Paris, IAU Île-de-France, 2008, G 3-3138

80 Quaternary glaciations in the Northern hemisphere : report no. 4 on the session in Stuttgart, September 1976, Prague, Geological Survey, 1977, G 3-3121

81 Réinventer le développement économique local, Paris, Conseil national des économies régionales, 2009, G 3-3123 

L'Harmattan, 2009, G 3-3130

\section{Dictionnaires}

\section{Europe} Mirton, 2008, G 15-6012

\section{G 15-6009} l'Aube, INED, 2009, G 15-6010 2005, G 15-6031 15-6049 2009, G 15-6023 Bourgogne, 2009, G 15-6045 L'Harmattan, 2009, G 15-6051 2009, G 15-6024

Sécurité et justice: enjeu de la politique extérieure de l'Union européenne, Bruxelles, Éd. de l'Université de Bruxelles, 2003, G 3-3132

SEMMOUD, Mers, détroits et littoraux: charnières ou frontières des territoires ?, Paris,

LAKEHAL, Dictionnaire de science politique: les 1500 termes politiques et diplomatiques pour rédiger, comprendre et répondre au discours politique, Paris, L'Harmattan, 2009, W 403

SAUNIER, MEGANCK,Dictionary and introduction to global environmental governance, London; Sterling, Earthscan, 2009, W 401

SFEIR, Dictionnaire géopolitique de l'islamisme, Montrouge, Bayard, 2009, W 402

AKÇALI, Chypre: un enjeu géopolitique actuel, Paris, L'Harmattan, 2009, G 15-6022

ALOMAR, Grandes questions européennes, Paris, Sedes, 2007, G 15-6034

ANCUŢA, Studiul geografic al disparităŢilor teritoriale din Banatul românesc, Timişoara, Ed.

BAS, CAYLA, L' Europe à la carte, Paris, Le Cherche midi, 2009, AQ 15-164

BAYOU, Itinéraires baltes : Estonie, Lettonie, Lituanie, Paris, Éd. Regards sur l'Est, 2005,

BONVALET, OGG, Les baby-boomers : une génération mobile, La Tour-d'Aigues ; Paris, Éd. de

BUFFOTOT, La défense en Europe : avancées et limites, Paris, La Documentation française,

CAMPANA, DUFAUD, TOURNON, Les déportations en héritage : les peuples réprimés du Caucase et de Crimée, hier et aujourd'hui, Rennes, Presses universitaires de Rennes, 2009, G

CANEROT, REY, Les Pyrénées: histoire géologique et itinéraires de découverte, Biarritz, Atlantica ; BRGM éd., 2008, G 15-6020

CARROUÉ, COLLET, RUIZ, L'Europe, Rosny-sous-Bois, Bréal, 2009, G 15-6032

CAUQUIL, Phénoménologie politique de l'Europe: l'union souveraine, Paris, L'Harmattan,

CHARLON, SARRÍA BUIL, Aragón : una identidad tierra adentro, Dijon, Université de

Chiffres clés de l'éducation en Europe 2009, Bruxelles, Agence exécutive Education, Audiovisuel et Culture, 2009, G 15-6039

01 CHILLAUD, Les îles Aland en mer Baltique : héritage et actualité d'un régime original, Paris,

2 Déchets municipaux en Europe : vers une société européenne du recyclage, Paris, Victoires éd.,

DUMONT, GÉRARDOT, L'Europe, Neuilly, Atlande, 2010, G 15-6011 
EDELBLUTTE, Paysages et territoires de l'industrie en Europe : héritages et renouveaux, Paris, Ellipses, 2010, G 15-6028

FRAGUAS Y FRAGUAS, Geografía de Galicia, Santiago de Compostela, Porto, 1953, G 15-6044

GAJDAR, La chute de l'empire soviétique : leçons pour la Russie d'aujourd'hui, Paris, EyrollesEd. D'organisation, 2010, G 15-6050

GARDE, Les Balkans : héritages et évolutions, Paris, Flammarion 2010, G 15-6052

GARNIER, Les dérangements du temps: 500 ans de chaud et de froid en Europe, Paris, Plon, 2010, G 15-6026

GUIU, TROCHET, Naissance d'une autre Catalogne : territoires et traditions dans les terres de l'Èbre, Paris, CTHS, 2008, G 15-6025

HAU, NARVAIZA SANTAMARIA, Le chômage en Europe : divergences nationales et régionales, Paris, Economica, 2009, G 15-6021

HERVOUET, Datcha blues : existences ordinaires et dictature en Biélorussie, Paris, Belin, 2009, G 15-6015

LEVÊQUE, RUIZ DEL ÁRBOL MORO, POP, Patrimoine, images, mémoire des paysages européens, Paris, L'Harmattan, 2009, G 15-6047

"L'identité européenne" : institutions et géopolitique : 2ème colloque, les 25 et 26 octobre 2008, Paris, Société de géographie, 2009, G 15-6017

JABKO, L'Europe par le marché: histoire d'une stratégie improbable, Paris, Presses de la fondation nationale de sciences politiques, 2009, G 15-6027

KLAIĆ,Mobility of imagination : a companion guide to international cultural cooperation, Budapest, Center for Arts and Culture, Central European University, 2007, G 15-6033

LAÏDI, La norme sans la force : l'énigme de la puissance européenne, Paris, Presses de Sciences Po, 2008, G 15-6014

LUSSAC, Géopolitique du Caucase : au carrefour énergétique de l'Europe de l'Ouest, Paris, Technip, 2009, G 15-6029

MANDOUL, Rotterdam, Paris, Cité de l'architecture et du patrimoine, IFA, 2009, G 15-6035

9 MAULIN, GROSSOUVRE, MOUSSON-LESTANG, L' Eurodistrict Strasbourg-Ortenau : la construction de l'Europe réelle, Konstruktion eines lebendigen Europa, Vevey (Suisse), Salde ; Xenia, 2009, G 15-6046

Le partenariat de l'espace européen de la recherche: initiatives 2008, Luxembourg, Office des publications officielles des Communautés européennes, 2009, G 15-6041

PASCO, L'Europe : dossier, Futuroscope, SCÉRÉN-CNDP, 2008, G 15-6038

PATTARONI, KAUFMANN, RABINOVICH, Habitat en devenir : enjeux territoriaux, politiques et sociaux du logement en Suisse, Lausanne, Presses polytechniques et universitaires romandes, 2009, G 15-6030

3 La politique de cohésion 2007-2013: commentaires et textes officiels: fonds européen de développement régional, fonds social européen, fonds de cohésion, groupement européen de coopération territoriale: instrument d'aide de pré-adhésion, Luxembourg, office des publications officielles des communautés européennes, 2007, G 15-6040 
RACZKA, WITT, Aux confins de l'Europe de l'Est : itinéraires entre la nostalgie et la révolte, entre la mémoire et l'espoir, Paris, L'Harmattan, 2009, G 15-6048

RADVANYI, KOURTOUBADZÉ, REKACEWICZ, Atlas géopolitique du Caucase : Russie, Géorgie, Arménie, Azerbaïdjan : un avenir commun possible ?, Paris, Autrement, 2009, G 15-6036

Resorts of national interest in the Romanian Carpathians : nature, local communities, tourist facilities, policies for sustainable development, Bucureşti, Ed. Universitară, 2009, G 15-6013

RUPNIK, Les banlieues de l'Europe: les politiques de voisinage de l'Union européenne, Paris, Presses de Sciences Po, 2007, G 15-6016

UNSTEAD,Europe, London, University of London Press, 1939, G 15-6043

URABAYEN, Una geografía de Navarra, investigación sobre las residencias humanas de Navarra, Pamplona, Libe, 1959, G 15-6042

\section{France}

ADOUMIÉ, Les régions françaises, Paris, Hachette Supérieur, 2010, G 10-5877

AUGRIS, CAILL-MILLY, CASAMAJOR, Atlas thématique de l'environnement marin du Pays basque et du sud des Landes, Versailles, Ed. Quae, 2009, G 10-5853

BARBAT-BUSSIÈRE, L'offre de soins en milieu rural: l'exemple d'une recherche appliquée en Auvergne, Clermont-Ferrand, Presses Universitaires Blaise Pascal, 2009, G 10-5870

BERTHIER, Plans climat-énergie territoriaux, Montreuil, Cédis; Réseau action climatFrance, 2009, G 10-5864

BILLEN, Le bassin de la Seine: découvrir les fonctions et les services rendus par le système Seine, Nanterre, Agence de l'eau Seine-Normandie, 2009, G 10-5844

BILLEN, GARNIER, Eutrophisation des cours d'eau du bassin de la Seine, S.l., s.n., 2009, G 10-5849

BOYER, L' hiver dans le Midi : XVIIe-XXIe siècles, Paris, L'Harmattan, 2009, G 10-5878

CARRÉ, BARBET-MASSIN, FRÊCHE, Montpellier: chroniques de Port Marianne, une histoire urbaine : 1989-2009, Paris, D. Carré, 2009, G 10-5859

CASTELLO, BOUCHI-LAMONTAGNE, Les eaux souterraines en France, Orléans, BRGM, 2009, G 10-5876

CHALINE, Les politiques de la ville, Paris, Presses universitaires de France, 2010, K 1078

CHARLOT-VALDIEU, OUTREQUIN, Écoquartier: mode d'emploi, Paris, Eyrolles, 2009, G 10-5855

CHEVREUIL, La micropollution organique dans le bassin de la Seine: maitriser l'impact des molécules créées par l'homme, Nanterre, Agence de l'eau Seine-Normandie, 2009, G 10-5852

CHIGNIER-RIBOULON, Les quartiers : entre espoir et enfermement, Paris, Ellipses, 2009, G 10-5866

Contribution au diagnostic du patrimoine de la commune d'Aulnay-sous-Bois, Pantin, Conseil Général Seine-Saint-Denis, 2008, G 10-5856

DAGUIN, De l'assiette aux champs, Paris, Direction des Journaux officiels, 2009, G 10-5889 
DARLEY, ZUNINO, PALISSE, Comment encourager l'intensification urbaine ?, Paris, IAU, Institut d'aménagement et d'urbanisme Île-de-France, 2009, G 10-5880

DEBRIE, LETILLEUL, La décentralisation portuaire : réformes, acteurs, territoires, Paris, L'Harmattan, 2010, G 10-5893

DUBOIS, Les politiques publiques territoriales: la gouvernance multi-niveaux face aux défis de l'aménagement, Rennes, Presses universitaires de Rennes, 2009, G 10-5867

L'eau urbaine, Paris, D. Carré, 2009, G 10-5860

Excursion "Hydrosystèmes anthropisés de la vallée de la Somme en Picardie" : 9-10-11 juillet 2007, Amiens, Université de Picardie-Jules Verne, 2007, G 10-5879

FASSIN, Les nouvelles frontières de la société française, Paris, La Découverte, 2010, G 10-5888

GUILLEMAIN, Quatre essais sur la notion de territoire dans le Centre de la France, Souvigny, Groupe de Souvigny, 2004, G 10-5884

HERVIEU, Les orphelins de l'exode rural : essai sur l'agriculture et les campagnes du XXIe siècle, La Tour-d'Aigues, Éditions de l'Aube, 2008, G 10-5895

LARUË-CHARLUS, Bordeaux Sud: Carle-Vernet, Belcier, Saint-Jean, Dormoy, Sainte-Croix, Capucins, Albert 1er, Ars, Nansouty, sacré-Coeur, Bordeaux, Overworld, 2009, G 10-5887

LEFÈVRE, SABARD, Les écoquartiers : l'avenir de la ville durable, Rennes, Apogée, 2009, G 10-5865

LEQUESNE, La France dans la nouvelle Europe, assumer le changement d'échelle, Paris, Presses de la fondation nationale des sciences politiques, 2008, G 10-5861

LE SAOUT, L'ntercommunalité en campagne : rhétoriques et usages de la thématique intercommunale dans les élections municipales de 2008, Rennes, Presses universitaires de Rennes, 2009, G 10-5863

LOMBARD-JOURDAN, Les Halles de Paris et leur quartier dans l'espace urbain (1137-1969), Paris, École Nationale des Chartes, 2009, G 10-5894

LUCA BARRUSSE, Démographie sociale de la France: XIXe-XXIe siècle, Paris, Presses universitaires de France, 2009, G 10-5857

MACHER, L' adolescence, une chance pour la ville, Paris, Les Carnets de l'info, 2010, G 10-5883

MANTEI, BOUYER, FAESSEL-VIROLE, Tourisme, urbanisme et aménagement sur le littoral et en montagne: les outils stratégiques et opérationnels pour une offre d'hébergement marchand, Paris, Atout France, 2009, G 10-5858

MOUHOUD, Economie des services et développement des territoires, Paris, La Documentation française, 2010, G 10-5885

PARIS, MONS, Lille métropole: laboratoire du renouveau urbain, Marseille, Parenthèses, 2009, G 10-5871

PENVEN, Ville et coopération sociale, Paris, L'Harmattan, 2010, G 10-5892

PITTE, Bordeaux Bourgogne: les passions rivales, Paris, Hachette Littératures, 2007, G 10-5862

Les quartiers durables: nouvel enjeu de la ville de demain ?, [S.l.], Observatoire de la ville, 2007, G 10-5882 

hydrodynamique du bassin pour une gestion durable de la ressource, Nanterre, Agence de l'eau Seine-Normandie, 2009, G 10-5845

177 VIENNOT, La pollution du bassin de la Seine par les nitrates: comprendre l'origine et la migration des nitrates dans l'écosystème pour mieux protèger les aquifêres, S.l., s.n., 2009, G 10-5846

\section{Généralités (méthode, épistémologie, histoire, cartographie, SIG)}

RAMEAU, BARDAT, KELLER, Flore forestière française: guide écologique illustré, Paris, Institut pour le développement forestier; Ministère de l'agriculture et de la pêche, Direction de l'espace rural et de la forêt ; Ecole nationale du génie rural des eaux et des forêts, 2008, G 10-5570-<3> durable de métropole, gouvernance et mémoires d'urbanité Talence, Bastingage, 2008, G 10-5886 10-5891 comprendre les enjeux de la gestion de l'eau, Nanterre, Agence de l'eau Seine-Normandie, 2009, G 10-5848

d'Algérie en métropole, 1954-2005, Paris, Éd. de l'École des hautes études en sciences sociales, 2010, G 10-5890 est contaminée pour déterminer les risques et les moyens à mettre en oeuvre pour restaurer sa qualité, Nanterre, Agence de l'eau Seine-Normandie, 2009, G 10-5851

SUBRA, Le Grand Paris, Paris, A. Colin, 2009, G 10-5872

TALES, Le peuplement de poissons du bassin de la Seine: comprendre son évolution sous l'impact des changements générés par l'homme ou la nature, Nanterre, Agence de l'eau Seine-Normandie, 2009, G 10-5847 circulent les métaux dans un bassin versant fortement exposé aux pressions humaines, Nanterre, Agence de l'eau Seine-Normandie, 2009, G 10-5850

ANIER, Le pouvoir des territoires : essai sur l'interterritorialité, Paris, Economica ; Anthropos, 2010, G 10-5896

Ville de Cannes : histoire d'espaces publics, [S.1.]; Cannes culture, 2010, G 10-5875

ANSELIN, REY,Perspectives on spatial data analysis, Berlin, Springer, 2009, G 5-1815

BAMBERGER, Le LCPC, acteur du génie civil durable, Paris, LCPC, 2009, G 5-1813

BATAILLON, ROBIC, Géographes génération 1930: à propos de Roger Brunet, Paul Claval, Olivier Dollfus, François Durand-Dastès, Armand Frémont et Fernand Verger, Rennes, Presses universitaires de Rennes, 2009, G 5-1797

Biographies d'enquêtes : bilan de 14 collectes biographiques, Paris, Institut national d'études démographiques (Ined), 2009, G 5-1796 
CANS, Tazieff: le joueur de feu, Paris, Sang de la terre, 2010, G 5-1809

CHRISS, MARJORIE,An introduction to charts and their use, Glasgow, Brown \& Ferguson, 1944, G 5-1812

DEPREST, Géographes en Algérie (1880-1950): savoirs universitaires en situation coloniale, Paris, Belin, 2009, G 5-1795

HINKS,Maps and survey, Cambridge, The University Press, 1944, G 5-1811

HUGUENOT, La géographie quelle histoire ! Les grands témoins racontent le Festival international de géographie de Saint-Dié-des-Vosges, Haroué, G. Louis, 2009, G 5-1798

LAFFLY, Approche numérique du paysage: formalisation, enjeux et applications, Paris, Publibook, 2009, G 5-1801

LANGLOIS, Simulation des systèmes complexes en géographie : fondements théoriques et applications, Paris, Hermes science publ. ; Lavoisier, 2010, G 5-1814

LEUNG, YEE, Knowledge discovery in spatial data, Dordrecht, Springer, 2010, G 5-1805

MAURICE, Petit manuel des levés à vue pour les reconnaissances militaires: au moyen de l'équerre-métrique, Perpignan, Rondony, 1877, G 5-1807

PITTE, Notice sur la vie et les travaux de Pierre George (1909-2006) lue le lundi 12 octobre 2009, Paris, Palais de l'Institut, 2009, G 5-1808

Progress in spatial analysis : methods and applications, Dordrecht, Springer, 2010, G 5-1806

REGGIANI, NIJKAMP, Complexity and spatial networks : in search of simplicity, Dordrecht, Springer, 2009, G 5-1804

ROTHENBERG,Presenting America's world : strategies of innocence in National geographic magazine, 1888-1945, Aldershot, Ashgate, 2007, G 5-1799

SONIS, HEWINGS, Tool kits in regional science : theory, models, and estimation, Dordrecht, Springer, 2009, G 5-1803

WOOD,Map-reading for schools, London, G.G. Harrap, 1944, G 5-1810

\section{Géographie humaine}

L'adaptation au changement climatique, Paris, Eska, 2009, G 9-7260

ALLEMAND, Sous la ville durable le génie urbain: rencontre avec les ingénieurs de l'EIVP, Paris, Éd. les Carnets de l'info, 2009, G 9-7204

L'Alliance atlantique 1949-2009, Paris, IFRI, 2009, G 9-7258

ASCHER, Métapolis ou L'avenir des villes, Paris, O. Jacob, 2010, G 9-7254

ASCHER, BOURDIN, L'âge des métapoles, La Tour d'Aigues, Éd. de l'Aube, 2009, G 9-7215

L'atlas des civilisations : 6000 ans d'histoire, 200 cartes : comprendre le présent à la lumière $d u$ passé, Paris, Société éd. du Monde ; Malesherbes publ., 2008, G 9-7232

Atlas géostratégique 2010 : Guerres et paix... Le monde vu par les diplomates, les militaires, les ONG, les marchés financiers... Paris, Areion, 2009, G 9-7284

BASSAND, KAUFMANN, JOYE, Enjeux de la sociologie urbaine, Lausanne, Presses polytechniques et universitaires romandes, 2007, G 9-7207

BARRAQUÉ, NAHRATH, Usages et régulations des eaux urbaines, Toulouse, Erès, 2010, G 9-7251 
BEAUJARD, BERGER, NOREL, Histoire globale, mondialisations et capitalisme, Paris, La Découverte, 2009, G 9-7244

BESSE, Le goût du monde : exercices de paysage, Arles, Actes Sud ; Versailles, ENSP, 2009, G 9-7225

BONNET, Entreprises et territoires, Paris, Ellipses, 2010, G 9-7245

BRAZ, Penser la mondialisation, Paris, Ellipses, 2009, G 9-7234

BROWN, MCLAFFERTY, MOON,A companion to health and medical geography, Chichester ; Malden, Wiley-Blackwell, 2010, G 9-7211

BUSSI, Un monde en recomposition: géographie des coopérations territoriales, Mont-SaintAignan, Publications des Universités de Rouen et du Havre, 2009, G 9-7240

BUTTON, Transport economics, Cheltenham, E. Elgar, 2010, G 9-7255

COULAUD, $L^{\prime}$ automoville : ville, automobile et mode de vie, Paris, L'Harmattan, 2010, G 9-7278

COUTARD, LÉVY, Ecologies urbaines, Paris, Economica ; Anthropos, 2010, G 9-7279

CRIQUI, Les États et le carbone, Paris, Presses universitaires de France, 2009, G 9-7235

CROZET, LAFOURCADE, La nouvelle économie géographique, Paris, La Découverte, 2009, G 9-7223

DANIC, Enfants et jeunes dans les espaces du quotidien, Rennes, Presses universitaires de Rennes, 2010, G 9-7267

DELORME, La ville autrement, Sainte-Foy, Presses de l'Université du Québec, 2005, G 9-7264

DJEFLAT, BOIDIN, Le développement durable: quelle appropriation par les pays en développement, Louvain-la-Neuve, De Boeck, 2009, G 9-7249

DIMINESCU, PASQUIER, 2010. Les migrants connectés : TIC, mobilités et migrations. Paris, La Découverte, G 9-7277

DOCKÈS, Le sucre et les larmes : bref essai d'histoire et de mondialisation, Paris, Descartes \& Cie, 2009, G 9-7224

DUFRÉNOT, SAND, LORENZI, Après la crise ? Les politiques économiques dans le monde, Paris, Economica, 2009, G 9-7228

DUNLOP, Les 100 mots de la géographie, Paris, Presses universitaires de France, 2009, K 1076

FOL, La mobilité des pauvres : pratiques d'habitants et politiques publiques, Paris, Belin, 2009, G 9-7203

FOUCHER, Les nouveaux (dés)équilibres mondiaux, Paris, La Documentation française, 2009, G 9-7243

FRANÇOIS, Après le pétrole, la nouvelle économie écologique: les alternatives végétales à l'or noir, Paris, Ellipses, 2009, G 9-7239

8 FROGER, GERONIMI, MÉRAL, Diversité des politiques de développement durable: temporalités et durabilités en conflit à Madagascar, au Mali et au Mexique, Paris, Karthala ; GEMDEV, 2009, G 9-7227

FROST, HALL, Tourism and national parks : international perspectives on development histories and change, Oxon ; New York, Routledge, 2009, G 9-7201 
GÉRARDIN, BROT, Économie de la connaissance et développement: quels transferts?, Louvain-la-Neuve, De Boeck, 2009, G 9-7210

GILBERT, HENRY, Comment se construisent les problèmes de santé publique, Paris, La Découverte, 2009, G 9-7229

GLEIZES, La fin des paysans n'est pas pour demain, La Tour-d'Aigues, Éd. de l'Aube, 2009, G 9-7253

GOUJON, PRIE, Les voyageurs de l'eau, Paris, Dunod, 2010, G 9-7282

HALL, LEW, Understanding and managing tourism impacts : an integrated approach, Oxon ; New York, Routledge, 2009, G 9-7200

HALLÉ, La condition tropicale : une histoire naturelle, économique et sociale des basses latitudes, Arles, Actes sud, 2010, G 9-7275

JACKS, The Rape of the Earth : a world survey of soil erosion, London, Faber and Faber, 1941, G 9-7268

KARLSSON, ANDERSSON, CHESHIRE, New directions in regional economic development, Dordrecht ; London, Springer, 2009, G 9-7202

KONG, O'CONNOR,Creative economies, creative cities : Asian-European perspectives, Dordrecht, Springer, 2009, G 9-7257

LACOSTE, Géopolitique : la longue histoire d'aujourd'hui, Paris, Larousse, 2009, G 9-7213

KONINCK, Les poids du monde: évolution des hégémonies planétaires, Québec, Presses de l'Université du Québec, 2006, G 9-7266

LASSERRE, BURTON, LE STRAT, Eaux et territoires: tensions, coopérations et géopolitique de l'eau, Sainte-Foy, Presses de l'Université du Québec, 2005, G 9-7262

Le monde en 2025: la montée en puissance de l'Asie et la transition socio-écologique, Luxembourg, Office des publications officielles des communautés européennes, 2009, G 9-7261

LEMARCHAND, Les zones économiques périphériques, Lille, Université des sciences et technologies de Lille, UFR de Géographie et d'Aménagement, 2009, G 9-7209

Lever les barrières: mobilité et développement humains, Paris, La Découverte, 2009, G 9-7230

Le monde en musiques, Grenoble, Glénat, 2009, G 9-7208

MARGUERITE, Géo-couleurs du monde, Vesoul, Chez l'auteur, 2010, G 9-7283

MASSIERA, De la croissance au développement durable, Paris, L'Harmattan, 2009, G 9-7273

MAUGARD, Regard sur la ville durable : vers de nouveaux modes de vie, Paris, Éd. CSTB, 2010, G 9-7280

MERLINO, La ville autrement : l'énergie des réseaux, Paris, Autrement, 2010, G 9-7286

Les mesures de la ville. Paris, Editions EHESS, 2009, G 9-7276

MOREAU DEFARGES, Introduction à la géopolitique, Paris, Ed. du Seuil, 2009, G 9-7216

MOREAU DEFARGES, La mondialisation, Paris, Presses universitaires de France, 2010, K 1080

MORINIAUX, Les mobilités, Paris, Sedes, 2010, G 9-7220

MOULAERT, La Logique sociale du développement territorial, Québec, Presses de l'université du Québec, 2008, G 9-7263 

AQ 9-76 9-7241 9-7238

PAPAGIÁNNIS, Un patrimoine à préserver : les zones humides méditerranéennes, Paris, Buchet Chastel, 2009, G 9-7272

PAQUOT, L'espace public, Paris, La Découverte, 2009, G 9-7236

Penser l'espace politique, Paris, Ellipses, 2009, G 9-7233

RICCIOTTI, HQE : les renards du temple, [S.l.], Al Dante, 2009, G 9-7252

ROUSSEAU, DURAND, KONINCK, Une seule terre à cultiver : Les défis agricoles et alimentaires mondiaux, Québec, Presses de l'Université du Québec, 2009, G 9-7265

SANNI YAYA, Le défi de l'accessibilité et de l'équité en santé dans le tiers-monde: entre droit fondamental, justice sociale et logique marchande, Paris; Québec, L'Harmattan ; Presses de l'Université Laval, 2009, G 9-7242

SASSI, AYCARD, VALLAUD, Atlas historique de la Méditerranée, Paris, Fayard; Beyrouth, Presses de l'université Saint-Joseph, 2009, G 9-7214

SHAW, World economic geography : with an emphasis on principles, New York ; London, Wiley ; Chapman \& Hall, 1955, G 9-7269

SCHMID,Economy of fascination: Dubai and Las Vegas as themed urban landscapes, Berlin, Borntraeger, 2009, G 9-7231

SÉNÉCAL, BHERER, La métropolisation et ses territoires, Québec, Presses de l'université du Québec, 2009, G 9-7206

SOPPELSA, Les 7 défis capitaux du nouvel ordre mondial : géopolitique du monde contemporain, Paris, A2C médias, 2009, G 9-7248

SPIRITO,Ecostructures : expressions d'une architecture durable, Vercelli, White Star, 2009,

THIBAUD, Systèmes de production et durabilité dans les pays du Sud, Paris, Khartala, 2009, G

THUAL, Le fait juif dans le monde: géopolitique et démographie, Paris, O. Jacob, 2009, G

THUREAU-DANGIN, Le monde en 2010 : les enjeux géopolitiques, les scénarios de sortie de crise, les prévisions pars pays et par secteurs, Paris, Courrier international, 2009, G 9-7256

TIMOTHY, NYAUPANE,Cultural heritage and tourism in the developing world, London, Routledge, 2009, G 9-7199

TIROLE, MISTRAL, SÈVE, Politique climatique: une nouvelle architecture internationale, Paris, La Documentation française, 2009, G 9-7237

TRAN, Les migrations assistées et forcées des Britanniques au XIXe siècle : l'identité ouvrière à l'épreuve de l'émigration, Paris, L'Harmattan, 2010, G 9-7274

TSAGUÉ, La pollution due au transport urbain et aéroportuaire: caractéristiques et méthodes de réduction, Paris, L'Harmattan, 2009, G 9-7247

TULIPPE, Cours de géographie humaine. Deuxième partie, Géographie humaine générale, Liège, Desoer, 1942-1945, G 9-7271

TULIPPE, Cours de géographie humaine. Première partie, Monographies synthétiques, Liège, Desoer, 1942-1945, G 9-7270

UZAN, CASSINGENA-TRÉVEDY, Les chemins de l'océan : océans et mondialisation, Aix-enProvence, Ed. du Gerfaut, 2009, G 9-7226 

2009, G 9-7250 2009, G 9-7246 2009, G 9-7205 G 8-3259

\section{Mélanges}

\section{Monde arabe} 2009, G 24-441

VALLAUX, Mers et océans, Paris, Les éd. Rieder, 1932, G 9-7281

VANDERMOTTEN, Géopolitique et populations, Villeneuve d'Ascq, Université de Lille 1,

VERNIER, Les énergies renouvelables, Paris, Presses universitaires de France, 2009, K 1077

VÉTILLARD, Énergie, climat, développement: l'heure des choix, Paris, L'Harmattan,

WACKERMANN, L'écosociété, Paris, Ellipses, 2010, G 9-7287

WACKERMANN, ROUGIER, L'eau : ressources et usages, Paris, Ellipses, 2010, G 9-7212

WIXTED,Innovation system frontiers : cluster networks and global value, Berlin, Springer,

\section{Géographie physique}

ARNDT, Ressources minérales : origine, nature et exploitation, Paris, Dunod, 2010, G 8-3261

KANDEL, KANDEL, La catastrophe climatique, Paris, Hachette Littératures, 2009, G 8-3255

MACCRACKEN, MOORE, TOPPING, Sudden and disruptive climate change : exploring the real risks and how we can avoid them, London; Sterling, Earthscan, 2008, G 8-3254

MALONE, Debating climate change : pathways through argument to agreement, London; Sterling, Earthscan, 2009, G 8-3253

MATHIEU, Les principaux sols du monde: voyage à travers l'épiderme vivant de la planète Terre, Paris, Tec \& Doc ; Lavoisier, 2009, G 8-3257

MERZERAUD, Stratigraphie séquentielle: histoire, principes et applications, Paris, Vuibert ; Société géologique de France, 2009, G 8-3256

NICOLLET, Métamorphisme et géodynamique : cours et exercices corrigés, Paris, Dunod, 2010,

RITTAUD, Le mythe climatique, Paris, Seuil, 2010, G 8-3260

SCHNEIDER, Les traumatismes de la terre: géologie des phénomènes naturels extrêmes, Paris, Vuibert ; Société géologique de France, 2009, G 8-3258

VALLIN, Du genre et de l'Afrique: hommage à Thérèse Locoh, Paris, Institut national d'études démographiques (Ined), 2009, G 6-347

ABDELMALKI, BEN SOLTANE, SADNI-JALLAB, Le Maghreb face aux défis de l'ouverture en Méditerranée, Paris, L'Harmattan, 2009, G 24-444

AKL, BEYHUM, Conquérir et reconquérir la ville: l'aménagement urbain comme positionnement des pouvoirs et contre-pouvoirs, Tripoli (Liban), Université de Balamand,

BEN HAMMOUDA, Émergence en Méditerranée : attractivité, investissements internationaux et délocalisations, Paris, L'Harmattan, 2009, G 24-443 


\section{Périodiques} 4586-8-3 3074-56 P 4719-2009 2009, P 1807-52 797-5304-05

KHALIFÉ, Le Liban: Phoenix à l'épreuve de l'échiquier géopolitique international, 1950-2008, Paris, L'Harmattan, 2009, G 24-442

MOUSSALIM, Contribution à l'évaluation des projets de développement rural local: le projet "Jessour et Ksour de Béni Khédache", Montpellier, CIHEAM-IAMM, 2009, G 24-445

Travail d'atelier : projet urbain à Gemmayzé, Beyrouth, ALBA, 2009, G 24-446

ANTOINE, GOLAZ, Vieillir au sud, Bondy, Institut de recherche pour le développement, 2010, P 1652/A-53

BABY-COLLIN, BENSAÂD, SINTES, Migrations et territoires de la mobilité en Méditerranée, Aix-en-Provence, Publications de l'Université de Provence, 2010, P 1420-113

BLUM, LEFÈVRE, SEBILLE, La famille d'Est en Ouest: un demi-siècle de transformations en Europe, Paris, A. Colin, 2009, P 3740-40-3/4

BONTE, BEN HOUNET, La tribu à l'heure de la globalisation, Paris, Éditions de l'École des hautes études en sciences sociales, 2010, P 1511-184

Catalogne, Catalognes, Toulouse, Presses universitaires du Mirail, 2009, P 460/A-28

Chine-Europe, fuis-moi je te suis, Paris, Choiseul, 2009, P 4741-20

Climate change as environmental and economic hazard, London, Earthscan Journals, 2009, P

COUBÈS, La frontière Mexique-Etats-Unis après 15 ans d'ALENA, Paris, IHEAL, 2009, P

DESRUES, HERNANDO DE LARRAMENDI, S'opposer au Maghreb : dossier, Paris, CNRS, 2009,

DEVILLET, Etre géographe aujourd'hui: la géographie....ma géographie: hommage au Professeur Bernadette Mérenne-Schoumaker, Liège, Éd. de la société géographique de Liège,

DRIANT, Les politiques du logement en France, Paris, La Documentation française, 2009, $\mathbf{P}$

France, enjeux territoriaux, Paris, La Découverte, 2009, P 3536-135

Eau et développement en Afrique tropicale : quelques expériences au Cameroun et au Burkina Faso, Toulouse : Université Toulouse-Le Mirail, 2010, P 3676-57

FELLOUS, Les droits de l'homme: une universalité menacée, Paris, La Documentation française, 2010, P 797-5306-07-08

14 Femmes et géopolitique, Paris, La Découverte, 2010, P 3536-136

FISCHER, LUZIA, Slope instabilities on perennially frozen and glacierised rock walls : multiscale observations, analyses and modelling, Zürich, Geographisches Institut der Universität Zürich, 2009, P 3881-58

FLEURANT, Paysage : approches qualitatives et quantitatives, Rennes, Presses universitaires de Rennes, 2010, P 1167-213

7 GALLINO, GAUCHON, L'hydrothermalisme en domaine continental: fonctionnement, ressource et patrimoine, Chambéry, Laboratoire EDYTEM, Université de Savoie, 2009, P 4608-9 
Géopolitiques japonaises, Paris, Institut international d'études stratégiques, 2010, P 4610-26

GRONDEAU, SCHIRRER, BURGEL, Le Grand Paris : matériaux pour une altermétropolisation, Paris, Laboratoire de géographie urbaine, 2008, P 3695/A-4

HIJMA,From river valley to estuary : the early-mid Holocene transgression of the Rhine-Meuse valley, The Netherlands, Utrecht, Koninklijk Nederlands Aardrijkskundig Genootschap, Faculteit Ruimtelijke Wetenschappen, Universiteit Utrecht, 2009, P 3619/B-389

Japon : un modèle à réinventer, Paris, La Documentation française, 2010, P 1102-2986

LAMAND, Ville, urbanisme \& tourisme, Paris, Editions touristiques européennes, 2010, P 4198-104

LÓCZY, DÉNES, KOVÁCS,Geomorphology in the Carpatho-Balkan-Dinaric Countries, Berlin, Gebrüder Borntraeger, 2009, P 10-53-2

MADORÉ, Gestion urbaine et inégalités socio-spatiales France-Brésil : varia, Rennes, Presses universitaires de Rennes, 2009, P 1167-212

MANNSFELD, SYRBE,Naturräume in Sachsen, Leipzig, Deutsche Akademie für Landeskunde, 2008, P 42-257

MIAUX, Corps urbains : mouvements et mise en scène, Paris, L'Harmattan, 2009, P 4350-70

MIOSSEC, Zones humides, chasse et conservation de la nature : actes du colloque tenu à la Maison de la baie de Somme et de l'oiseau, 17-19 juin 2009, Cordemais, Estuarium, 2010, P 4613 [16]

MOISSERON, Action publique et gouvernance en Méditerranée, Paris, Choiseul, P 4532-202

PAPE,Predictability of nearshore sandbar behavior, Utrecht, Koninklijk Nederlands Aardrijkskundig Genootschap, Faculteit Geowetenschappen Universiteit Utrecht, 2010, P 3619-392

Planification Lyon (1984) 2010 : dossier, Paris, Les Publications d'architecture et d'urbanisme, 2010, P 431-371

RAULIN, KUCZYNSKI, Anthropologie et migrations : parcours et recherches, Poitiers, Association pour l'étude des migrations internationales, 2009, P 4045-25-3

Reconfigurations religieuses en République populaire de Chine, Hong-Kong, CEFC, 2009, P 4681-4

SCHULTZ, Brain drain aus Ostdeutschland?: Ausmass, Bestimmungsgründe und Folgen selektiver Abwanderung, Leipzig, Deutsche Akademie für Landeskunde, 2009, P 42-258

SCHNEIDER-SLIWA, SAALFRANK,Local adaptive capacities and co-management in disaster risk reduction : targeted analyses in local communities of Gujarat and Tamil Nadu : technical report for the funding agencies, Basel, Department of Geography, University of Basel, 2009, P 4788-2

SELLIN, MICHON, Zones urbaines partagées, Saint-Denis, Synesthésie, 2008, P 4791-2

Sud-Ouest de l'Océan Indien, Pessac, Presses universitaires de Bordeaux, 2009, P 945-248-62

Tourisme, sport et développement, Montréal, Université du Québec, 2009, P 4703-28-2

Travail à bas salaire en Europe et aux Etats-Unis, Genève, Bureau International du Travail, 2009, P 1573-148-4 

4350-71

\section{Thèses}

VAN SCHAIK,The role of macropore flow from PLOT to catchment scale : a study in a semi-arid area, Utrecht, Koninklijk Nederlands Aardrijkskundig Genootschap, Faculteit Ruimtelijke Wetenschappen, Universiteit Utrecht, 2010, P 3619/B-390

ZENEIDI-HENRY, Où en est la rue face à la globalisation ?, Paris, L'Harmattan, 2010, P

\section{Ressources électroniques}

ARTHUS-BERTRAND, Home, Paris, Europacorp ; Elzevir films, 2009, DVD 103

BURKEL, Pain, pétrole et corruption, Paris, Doc et film international ; ADAV, 2009, DVD 95

CAUCHARD,Made in China, Paris, Ampersand, 2009, DVD 101

LANOY, Alerte aux pôles, Meudon, CNRS Images, 2008, DVD 99

LEVAIN, Herbe, Paris, Blaq out, 2009, DVD 94

LUNETTA, Les nouveaux tourismes, Paris, ADAV ; Doc\&film, 2010, DVD 96

MOREIRA, Afghanistan : sur la piste des dollars, Paris, ADAV ; Doc \& Co, 2007, DVD 97

RABHI, A la découverte de l'agriculture biologique, Paris, Parallele, 2009, DVD 104

WAGENHOFER,Let's make money, Paris, Ad vitam ; MK2, 2009, DVD 100

YUNG,Up the Yangtze, Paris, BQHL, 2009, DVD 102

ETIENNE, Aléas climatiques et croissance démographique : le cas de la ville des Gonaïves, [S.l.] [s.n.], 2009, D 2737

GRIVEL, GAUTIER, GILLI, La Loire des îles, du Bec d'Allier à Gien : rythme d'évolution et enjeux de gestion, S.l., s.n., 2008, T 2068 